\title{
Pretreatment technology of lignocellulose
}

\author{
Guoming Zeng ${ }^{1,2,3}$, Sijie $\mathrm{He}^{1}$, Yan $\mathrm{Li}^{5, \mathrm{a}^{*}}$, Da Sun ${ }^{4}$, Haonan $\mathrm{Li}^{1}$, Xin Wen ${ }^{1}$, Jun Wang ${ }^{6, b^{*}}$ \\ ${ }^{1}$ Chongqing University of Science and Technology, Chongqing 401331, China \\ ${ }^{2}$ China Metallurgical Construction Engineering Group Co., Ltd., Chongqing 643000, China \\ ${ }^{3}$ Chongqing University, Chongqing 40044, China \\ ${ }^{4}$ Wenzhou University, Wenzhou 325035, China \\ ${ }^{5}$ School of Pharmacy, Taizhou polytechnic college, Taizhou 225300, China \\ ${ }^{6}$ CCTEG CHONGQING ENGINEERING(GROUP) CO., LTD., Chongqing 643000, China
}

\begin{abstract}
Lignocellulose is the most abundant renewable biomass resource in nature. Pretreatment of lignocellulose can improve the accessibility of cellulase to cellulose raw materials, reduce the ineffective adsorption of cellulase, reduce the crystallinity and obtain higher reducing sugar. In this paper, several practical pretreatment technologies of lignocellulose are summarized, and the methods, principles, advantages and disadvantages of each pretreatment technology are summarized, and then the development prospect of lignocellulose pretreatment methods is prospected.
\end{abstract}

\section{Introduction}

With the depletion of non-renewable energy, the research and development of emerging energy is receiving more and more attention from all countries. Among them, lignocellulose is widely used in the production of ethanol, lactic acid, bio-oil and other products. Because of its abundant sources, low prices, and easy industrial production, research on lignocellulose has gradually increased in various countries.

However, due to the complex structure of lignocellulose raw materials, and the cellulose is wrapped in lignin and hemicellulose, coupled with the dense crystalline structure of cellulose itself, the efficiency of cellulose enzymatic hydrolysis is very low, which is not conducive to large-scale industrial production and lignocellulosic must be pretreated. The lignocellulose raw materials mainly contain cellulose, hemicellulose and lignin. The composition of several important lignocellulose raw materials is shown in Table 1, in which the amorphous region of cellulose is surrounded by hemicellulose, and the lignin is surrounded by crystallization zone. The schematic diagram of the structure of lignocellulose is shown in Figure 1. Therefore, to increase the yield of reducing sugars by enzymatic hydrolysis of cellulosic enzymes, the enveloping effect of hemicellulose and lignin must be removed.

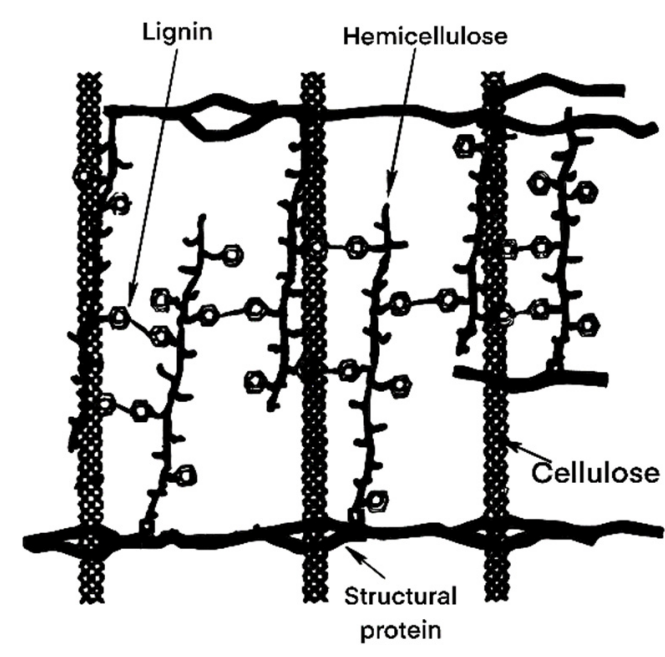

Fig 1. Schematic diagram of lignocellulose structure

Table1. The composition of several important lignocellulosic raw materials

\begin{tabular}{cccc}
\hline $\begin{array}{c}\text { Lignocellul } \\
\text { osic raw } \\
\text { materials }\end{array}$ & $\begin{array}{c}\text { Cellulose } \\
(\%)\end{array}$ & $\begin{array}{c}\text { Hemicellulose } \\
(\%)\end{array}$ & $\begin{array}{c}\text { Lignin } \\
(\%)\end{array}$ \\
\hline hardwood & $40 \sim 55$ & $24 \sim 40$ & $18 \sim 25$ \\
cork & $45 \sim 50$ & $25 \sim 35$ & $25 \sim 35$ \\
Corn stalks & 40 & 25 & 17 \\
Corn cob & 45 & 35 & 15 \\
Wheat straw & 30 & 50 & 20 \\
Rice straw & 35 & 25 & 12 \\
Bagasse & 40 & 24 & 25
\end{tabular}

\footnotetext{
* Corresponding author: a55397507@qq.com

b247215325@qq.com
} 


\begin{tabular}{cccc} 
grass & $25 \sim 40$ & $35 \sim 50$ & $10 \sim 30$ \\
leaf & $15 \sim 20$ & $80 \sim 85$ & 0 \\
\hline
\end{tabular}

The main purpose of pretreatment is improving the accessibility of cellulase to cellulosic raw materials, reducing the ineffective adsorption of cellulase, reducing crystallinity, and obtaining higher reducing sugars. Before lignocellulose is used, it needs to go through the pretreatment stage to better ferment sugar production [2]. The current pretreatment can be divided into physical method, chemical method, biological method, physical chemical method and so on.

\section{Materials and methods}

\subsection{The purpose of lignocellulose pretreatment}

Lignocellulose includes three main components: cellulose, hemicellulose and lignin. As an important component of plant cell wall, lignocellulose plays a role in protecting cells. Lignin is a high molecular aromatic compound formed by phenylpropane and its derivatives, which plays a barrier role in the hydrolysis of cellulose. Therefore, the presence of lignin hinders the contact of enzymes with cellulose and hemicellulose, and the combination of lignin with hydrolase is irreversible, which leads to the decrease of hydrolysis rate.

Pretreatment of lignocellulose refers to the destruction of the high crystallinity structure of lignocellulose by certain technical means, which makes it easy to be further degraded and utilized. Pretreatment can destroy the wrapping of lignin and hemicellulose on cellulose, remove lignin, degrade hemicellulose, change the crystal structure of cellulose, increase the accessible inner surface area and loose degree of cellulose, increase the reaction area between enzyme and substrate, and significantly increase the subsequent enzymatic hydrolysis efficiency and sugar yield. At present, lignocellulose pretreatment processes can be divided into four categories; Physical method, chemical method, biological method, physical and chemical method, and the selection of specific methods depends on materials.

\subsection{Pretreatment method}

\subsubsection{Physical method}

Physical methods mainly reduce the particle size and crystallinity of lignocellulose directly through traditional mechanical grinding or radiation, mainly including mechanical crushing, ultrasonic and microwave treatment, etc.

\subsubsection{Chemical method}

Commonly used chemical pretreatment methods mainly include acid treatment, alkali treatment, organic solvent treatment, ozone treatment and so on.

\subsubsection{Biological method}

Biological method is to use fungi to degrade lignocellulosic materials and achieve the purpose of removing the adhesive layer package. In the biological pretreatment method, some microbial species such as white rot fungi, soft rot fungi and brown rot fungi are widely used to degrade hemicellulose and lignin.

\subsubsection{Physical and chemical method}

The main physical and chemical methods are steam explosion and ammonia explosion.

\subsubsection{Chemical combination pretreatment method}

This method is a new type of pretreatment method. The treatment method has relatively mild treatment conditions, low equipment requirements, and low energy consumption. There are mainly two combinations of strong acid-strong base (dilute sulfuric acid-sodium hydroxide) or weak acid-weak base (formic acid-ammonia)

\subsubsection{Green solvent pretreatment method}

The research shows that NMO, as an industrial cellulose solvent, can treat lignocellulose with high crystallinity.

\subsubsection{Plasma pretreatment method}

Plasma pretreatment mainly uses electric energy to form highly active ionized gas to destroy the complex structure of lignocellulose. According to different media properties, it can be divided into air (nitrogen), ozone and argon plasma pretreatment methods.

\section{Results and discussion}

\subsection{Physical method}

Mechanical pulverization refers to reducing the particle size of lignocellulosic materials through milling, shearing, and increasing the specific surface area of the materials, thereby effectively reducing the crystallinity of cellulose and destroying the binding layer of hemicellulose and lignin which is a more common pretreatment method. Due to the decrease of cellulose crystallinity, the water-soluble components of the material are increased, thereby increasing the yield of cellulose hydrolyzed reducing sugars. However, the mechanical pulverization method cannot change the composition of cellulose raw materials and the crystal structure of cellulose. Therefore, the mechanical pulverization method can only improve the hydrolysis to a limited extent.

Ultrasound is a sound wave with a frequency of $2 \times 10^{4}$ $\mathrm{Hz}$ to $10^{9} \mathrm{~Hz}$, which is fast, efficient, and pollution-free. It treats lignocellulosic raw materials through ultrasonic cavitation, which generates stress in the fiber voids and causes microcrystalline dislocations, thereby increasing the specific surface area of the cellulose materials, 
increasing the amorphous area, and reducing the cellulose crystallinity. Studies have found that ultrasonic waves can not only effectively destroy the hydrogen bonds in and within the cellulose molecules, but also effectively reduce its regularity and crystallinity. However, the effect of ultrasound on the fine structure of cellulose has certain limitations, and due to the degradation of hemicellulose, the specific surface area of cellulose has decreased, which is extremely unfavorable for subsequent enzymatic hydrolysis of cellulose. Microwave is an electromagnetic wave whose wavelength is in the range of $1 \mathrm{~mm}$ to $100 \mathrm{~cm}$. It has the characteristics of short use time, convenient operation, energy saving and environmental protection, and high saccharification efficiency.

Microwave treatment can generate heat energy from cellulose molecules to reduce the crystallinity of cellulose and increase the enzymatic hydrolysis rate. Microwave pretreatment alone has a certain effect on the enzymatic hydrolysis of lignocellulose raw materials, but high temperature can also cause problems with a large amount of loss of value components. Therefore, further research is needed to improve.

\subsection{Chemical method}

Acid treatment has the advantages of low catalytic cost, easy to be neutralized by alkali, and high yield of reducing sugar obtained by hydrolysis of hemicellulose. However, the five-carbon sugar, which is the hydrolysate of hemicellulose after acid treatment, is prone to produce a large amount of furfural, which inhibits the subsequent fermentation of ethanol and other products.

Alkaline treatment mainly degrades the lignin in lignocellulose, and ultimately leads to the decrease of cellulose crystallinity. Alkaline treatment mainly includes ammonia treatment and sodium hydroxide treatment. It mainly reduces the lignin content in the lignocellulosic raw material and breaks the space structure between lignin, cellulose and hemicellulose, thereby improving the hydrolysis efficiency of cellulase. However, this method has higher requirements for reaction equipment and has a greater environmental pollution.

Oxidation treatment uses gases such as ozone to increase the enzymatic hydrolysis rate of cellulose. However, the production cost of this method is relatively high, and it is difficult to realize large-scale industrial production.

Organic solvent treatment refers to the separation of lignin from lignocellulose raw materials. One solvent or a combination of multiple solvents can be used to change the structure of lignocellulose and improve the efficiency of enzymatic hydrolysis. After the method, the organic solvent is difficult to recycle, and the loss of hemicellulose is large, which is not conducive to the comprehensive utilization of lignocellulosic raw materials.

\subsection{Biological method}

The advantages of biological method are mild environmental conditions, strong specificity of bacteria, energy saving and environmental protection. The disadvantage is that the current treatment time of this method is long, the microbial growth conditions are strict, and it only stays in the laboratory research and development stage, it is difficult to apply to industrial production, and there are fewer types of lignin-degrading microorganisms and the enzyme activity of lignindecomposing enzymes ${ }^{[3,5]}$. Low, long processing time, so the genetic improvement of white rot fungi and other bacterial species is helpful to the practical application of biological methods.

\subsection{Physical and chemical method}

Steam explosion treatment refers to the treatment of lignocellulosic materials under high temperature and high pressure with water or steam, and then suddenly reduces the steam pressure, which leads to the destruction of the internal spatial structure of lignocellulose, it is not only reduces the crystallinity of cellulose, but also reduces the enveloping effect of hemicellulose on cellulose. However, this method has a large loss of hemicellulose, and has a small effect on the components of lignin and cellulose, and has a high cost, which has certain limitations in industrial production.

The principle of ammonia explosion pretreatment is similar to that of steam explosion pretreatment. The difference is that the material needs to be soaked in ammonia water beforehand, and then steam explosion pretreatment, which can reduce the wrapping effect of the bonding layer of lignocellulosic material on the cellulose. Compared with the prior art, it can effectively remove lignin and destroy the dense crystalline structure of cellulose, thereby improving the accessibility of cellulase. The disadvantage is that the equipment cost and energy consumption of ammonia recovery are relatively high, and the price of ammonia itself is relatively expensive, which makes the cost of ammonia fiber blasting method high.

In the $\mathrm{CO}_{2}$ blasting treatment, the degradation of hemicellulose is accomplished by carbonic acid, and there is no by-product that inhibits subsequent fermentation in the treated lignocellulosic raw material.

\subsection{Chemical combination pretreatment method}

Most lignin was not removed after simple acid reaction of lignocellulose, which significantly affected the subsequent enzymatic efficiency ${ }^{[1]}$. However, after the combined reaction of acid and base, most non-cellulose substances were removed, and the inhibitors produced in the acid reaction process were greatly reduced, and the enzymatic efficiency was greatly increased. Lignin and hemicellulose were effectively removed from lignocellulose materials by strong acid-strong base (dilute sulfuric acid-sodium hydroxide ) or weak acid-weak base ( formic acid-ammonia ), resulting in a large number of cellulose substrates running. Formic acid treatment can cause cellulose accumulation, so the accessible surface area of the enzyme is significantly reduced, which reduces the enzymatic efficiency network. However, the hydrolysis efficiency was significantly increased with the increase of enzyme accessibility to the contact surface 
through subsequent ammonia immersion treatment in the mild system. Especially after the substrate was pretreated by acid-base combination, due to the increase of crosslinking degree, a small amount of non-cellulose materials were contained, and the adhesion was reduced under the same conditions, which could provide more favorable conditions for subsequent high solid reaction.

\subsection{Green solvent pretreatment method}

At high concentrations of NMO, not only the hydrogen bonds between the cellulose chains are completely broken, but the van der Waals forces between the cellulose chains are also weakened, and the cellulose crystallinity is significantly reduced. Moreover, NMO can also be recycled through biodegradation, is green and environmentally friendly, and has mild processing conditions and high efficiency. It is a new pretreatment technology with certain potential and application value.However, the efficiency and cost of scale-up and industrial production of this technology are not clear, which can not be widely used. Finding more new green pretreatment solvents and expanding large-scale production may be the problems to be solved by researchers in the future.

\subsection{Plasma pretreatment method}

Compared with other treatment processes, this method is simple to operate, green and pollution-free, and can also improve the efficiency of subsequent enzyme treatment.

\section{Conclusion}

In recent years, the research on lignocellulosic biomass energy has been highly valued and widely concerned by scholars, and a series of utilization and development have been carried out. At present, the lack of basic theoretical research on pretreatment limits the development of this technology ${ }^{[4]}$. It is necessary to further strengthen the research on the chemical composition of lignocellulose and the characteristics of substrate resistance to enzymatic hydrolysis, and analyze the reaction mechanism that affects the degradation of lignocellulose in a targeted manner, so as to provide information support for continuing to explore new pretreatment technologies of lignocellulose.

\section{Acknowledgments}

This work was supported by the National Natural Science Foundation of China (Grant No 51808086), China Postdoctoral Science Foundation (Grant No 2020M673112), Taizhou Science and Technology Support Program (Agriculture) Project (TN202012), Wenzhou Science and Technology Bureau Project (Grant No S2020005) and Provincial and Ministerial Coconstructive of Collaborative Innovation Center for MSW Comprehensive Utilization.

\section{References}

1. Mahmoodi, P., Karimi, K. and Taherzadeh, M.J. (2018) Efficient conversion of municipal solid waste to biofuel by simultaneous dilute-acid hydrolysis of starch and pretreatment of lignocelluloses[J]. Energy Conversion and Management, 166(JUN.):569-578.

2. Chen, H., Liu, J., Chang, X., Chen, D., Xue, Y., Liu, P., Liu, H. and Han, s. (2017) A review on the pretreatment of lignocellulose for high-value chemicals. Fuel Processing Technology, 160(Complete): 196-206.

3. Gao, D., Chundawat, S., Sethi, A., Balan, V., Gnanakaran, S. and Dale, B.E. (2013) Increased enzyme binding to substrate is not necessary for more efficient cellulose hydrolysis. Proceedings of the National Academy of Sciences of the United States of America, 110(27):10922-10927.

4. Prasad, A., Sotenko, M., Blenkinsopp, T and Coles, S.R. (2016) Life cycle assessment of lignocellulosic biomass pretreatment methods in biofuel production. The International Journal of Life Cycle Assessment, 21(1):44-50.

5. Satari, B., Karimi, K. and Zamani, A. (2016) Oil, chitosan, and ethanol production by dimorphic fungus Mucor indicus from different lignocelluloses[J]. Journal of Chemical Technology \& Biotechnology, 91(6):1835-1843. 